\title{
Evaluation of Novel co-processed excipient for fast disintegration of aspirin tablet formulations
}

\author{
Olubunmi J. OLAYEMI ${ }^{*}$ and Sophie NOCK-ANYEBE ${ }^{2}$ \\ ${ }^{I}$ Department of Pharmaceutical Technology and Raw Materials Development, National Institute for Pharmaceutical \\ Research and Development (NIPRD), Idu Industrial Area, Abuja. Nigeria. \\ ${ }^{2}$ Department of Pharmaceutics and Industrial Pharmacy, Ahmadu Bello University, Zaria. Nigeria.
}

Received $11^{\text {th }}$ September 2020; Accepted $3^{\text {rd }}$ November 2020

\begin{abstract}
Co-processing is a technique that ensures sub-particulate interaction of individual excipients leading to overall functionality of the resulting excipient. The aim of this study is to co-process Cyperus esculentus starch with mannitol by fusion and evaluate its effect on tablet disintegration and in vitro dissolution. Co-processed excipients were prepared from Cyperus esculentus starch and mannitol by fusion in ratios of 1:1, 1:2 and 2:1 (CM1, CM2, and CM3 respectively) and evaluated for flow and swelling properties. The excipients were incorporated into Aspirin tablet formulations at $5 \%$ w/w by direct compression (FM1, FM2, FM3 respectively). Similar tablets were prepared using sodium starch glycolate (FSG) and the formulations were assessed for hardness, friability, wetting time, disintegration time and in vitro dissolution profile. All the prepared excipients possessed excellent flow with Carr's index between 17.31 and 20.78 and Hausner ratio between 1.21 and 1.26. CM3 had the highest swelling profile (1.491) while CM2 had the lowest (1.321). Formulation FM1 had the highest tensile strength $\left(14.12 \mathrm{~N} / \mathrm{cm}^{2}\right)$ but slower wetting time (34.33 sec) compared to FM3 with tensile strength of $11.32 \mathrm{~N} / \mathrm{cm}^{2}$ and wetting time of $9.00 \mathrm{sec}$. Disintegration time of CM3 (4.26 min) was comparable to that of FSG (4.01 min); their dissolution profile was also found to be similar. Coprocessing Cyperus esculentus starch and mannitol by fusion (2:1) influenced tablet disintegration and in vitro dissolution and has potential to be used in manufacture of fast dissolving tablet formulations.
\end{abstract}

Keywords: Co-processing; Cyperus esculentus starch; Mannitol; Disintegration; Dissolution

\section{INTRODUCTION}

Excipients play a major role in the activity of an active ingredient viz-a-viz its delivery, safety and the overall cost of a product. Although a wide range of excipients exist currently, there is need for research scientists and formulators to develop excipients with specific characteristics to meet the requirements of the new drug moieties with varying physicochemical and stability properties [1].
A valuable means of improving excipient functionality is by co-processing. This concept is based on sub-particle interaction of two or more already existing excipients through appropriate techniques to obtain materials with improved properties over the individual excipients [2]. Co-processed excipients are popular for direct compression which gives added economic value to their use in product formulation. This process ensures that individual excipients interact at sub-particle

*Correspondence. E-mail: olubunmibiala@yahoo.co.uk Tel: +234-803 3532299.

ISSN 0189-8442 (cc) BY-NO 2021. Published by Faculty of Pharmaceutical Sciences, University of Jos, Nigeria. Under Creative Commons Attribution-Non-Commercial 4.0 International License. https://creativecommons.org/licenses/by-nc/4.0/ 
level leading to improved overall functionality of the new excipient. Specific benefits of coprocessed excipients include improved flow, better dilution potential, reduced sensitivity to lubricant effects, improved compressibility as well as masking of undesirable properties of individual excipients [3,4]. Co-processed excipients involve a combination of a plastic and brittle material to produce a complimentary effect in terms of flowability and compressibility [5]. An example of such plastic material is starch; it is one of the most versatile excipients used in tablet formulations; it is used as binder, diluent, disintegrant, glidant [6] and due to its availability in locally available plant sources, its various forms (modified or not) are being exploited to be used as substitute for the known and commercially available pharmaceutical starches. Starch from various sources has been co-processed with a variety of other excipients to produce multifunctional excipients with diverse applications [7-15). Mannitol is a brittle material [16] used as an excipient for lozenges, orodispersible and chewable tablet formulations because they provide good mouth feel, sweetness and are also used as filler in direct-compression tablet formulations $[17,18]$. Its non-hygroscopic nature has also been exploited as an advantage in the stability of tablet formulations.

In this study, starch from an unconventional source (Cyperus esculentus roots) also known as tiger nut, is used. Tiger nut starch has been exploited for use as binder, filler, disintegrant, suspending agent and coating agent [19-21]. Like all other native starches, tiger nut starch in its native form possesses poor flow and is poorly compressible but mannitol on the other hand possesses good compatibility. Consequently, combination of both materials (starch and mannitol) would result in a product with improved flowability, compatibility and dissolution.
Therefore, the aim of this study is to coprocess starch from Cyperus esculentus roots with mannitol in different ratios $(1: 1,1: 2$ and $2: 1)$. The effect of the produced excipients incorporated at $5 \% \mathrm{w} / \mathrm{w}$ on disintegration time and in vitro release of aspirin tablet formulations was evaluated.

\section{EXPERIMENTAL METHODS}

Materials. Aerosil (Evonik Industries, Japan), Mannitol (Sigma Aldrich, USA), Microcrystalline cellulose (BDH chemicals Ltd), Cyperus esculentus starch extracted in the National Institute for Pharmaceutical Research and Technology, NIPRD, Abuja, Nigeria

Extraction of starch. Cyperus esculentus starch was extracted using the described method [22] with some modifications. Fresh tiger nut roots $(1 \mathrm{~kg})$ were washed in water and soaked in $1 \% \mathrm{w} / \mathrm{w}$ sodium metabisulphite solution $(5 \mathrm{~L})$ at room temperature for $24 \mathrm{~h}$. Then the roots were removed from the solution, wet-milled into a slurry using a domestic milling machine. The slurry was strained through a calico cloth, the sediments were discarded while the filtrate was allowed to stand for $12 \mathrm{~h}$. Afterwards, the supernatant was discarded and the sediment starch was resuspended in water and centrifuged at 1500 rpm for $30 \mathrm{~min}$ in the centrifuge (Heraeus Sepatech Labofuge Ae, GmbH Germany). The supernatant was discarded and settled starch was placed on a tray, air-dried for $12 \mathrm{~h}$ and then dried in the oven at $40^{\circ} \mathrm{C}$ for $2 \mathrm{~h}$. The dried starch was pulverized in a mortar and then packaged for further analysis.

Co-processing of Cyperus esculentus starch and mannitol. The fusion method according to Alebiowu and Adeoye [10] was used with some modification. Appropriate quantities of Cyperus esculentus starch was suspended in water according to the compositions in Table 1 to obtain $40 \% \mathrm{w} / \mathrm{w}$ starch dispersion. This was stirred over a water bath at $50^{\circ} \mathrm{C}$ for $5 \mathrm{~min}$ to 
form a paste. Mannitol was incorporated into the paste by stirring over the water bath until homogenous, the paste was transferred unto a stainless tray and dried in the oven at $40^{\circ} \mathrm{C}$ for $2 \mathrm{~h}$. The dried mass was size-reduced in a mortar, passed through a sieve mesh size of $250 \mu \mathrm{m}$, packaged in a suitable container and kept in the desiccator until further use.

\section{Evaluation of co-processed excipients}

Flow rate. The time taken for the co-processed excipient $(5 \mathrm{~g})$ to flow through a funnel was noted. The glass funnel was fixed such that its tip was fixed at a height of $10 \mathrm{~cm}$ from a flat surface was noted. The flow rate $(\mathrm{g} / \mathrm{sec})$ was calculated as the ratio of the powder weight to the time taken for the powder to flow through the funnel.

Angle of repose. The funnel method was used [23]; five (5 g) of the co-processed excipient was poured into a funnel whose orifice had been plugged. The height and diameter of the powder heap formed after opening the orifice were determined and the angle of repose (A) was calculated from the equation;

$$
A=\tan ^{-1} \text { heightof heap } / \text { radius of heap ...Eqn } 1
$$

Bulk and tapped densities. The volume occupied by the co-processed excipient $(5 \mathrm{~g})$ in a measuring cylinder $(100 \mathrm{~mL})$ was recorded as the bulk volume. The measuring cylinder was tapped in the Stampfvolumeter (STAV 2003JEF, Germany) and the volume after tapping was recorded as the tapped volume. Bulk and tapped densities were calculated as shown below;

$$
\begin{aligned}
& \text { Bulk density }(\mathrm{g} / \mathrm{mL}) \\
& =\text { weight of powder } / \text { bulk volume ... Eqn } 2 \\
& \text { Tapped density }(\mathrm{g} / \mathrm{mL}) \\
& =\text { weight of powder } / \text { tapped volume } \ldots \text { Eqn } 3
\end{aligned}
$$

Hausner ratio (HR) and Compressibility Index $(C I)$. These were calculated from data obtained from the bulk and tapped densities;

$$
H R={ }^{\text {tapped density } / \text { bulk density } \quad . . . E q n ~} 4
$$

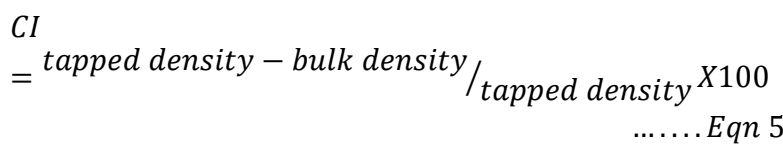

Moisture content. This was determined using the moisture analyzer (Ohaus MB 45, USA) fitted with an infrared heating unit. Five (5 g) of the co-processed excipient was placed into the analyzer set at drying temperature of $105^{\circ} \mathrm{C}$ for $15 \mathrm{~min}$ and percentage of moisture was obtained automatically.

Swelling power. A dispersion of the coprocessed excipient in water $(1 \% \mathrm{w} / \mathrm{v})$ was made in a beaker, this was heated for $30 \mathrm{~min}$ in a water bath thermostated at $37^{\circ} \mathrm{C}$ while stirring intermittently. The dispersion was centrifuged at $1500 \mathrm{rpm}$ for $30 \mathrm{~min}$ in the centrifuge (Heraeus Sepatech Labofuge Ae, $\mathrm{GmbH}$ Germany), the supernatant was decanted and the weight of the wet mass was determined. Swelling power (SP) was computed using the equation below;

$$
\begin{aligned}
& S P(\%)= \\
& \text { weight of wet mass/weight of dry powder X } 100
\end{aligned}
$$

This method was also used to determine swelling power of the co-processed excipient at $40,50,60,7080$ and $90^{\circ} \mathrm{C}$.

Preparation of aspirin tablets using the coprocessed excipients. The direct compression method was used in producing a batch of 30 tablets. Appropriate quantities of the coprocessed excipients at concentration of $5 \%$ $\mathrm{w} / \mathrm{w}$ were mixed with aspirin powder according to the composition in Table 2. Aspirin powder was weighed into the mortar then the diluent; microcrystalline cellulose (MCC) and glidant; aerosil were added into the content of the mortar in geometric measures and triturated together so as to ensure a homogenous powder mix. Another batch containing a reference superdisintegrant; sodium starch glycolate (SSG) at the same concentration was also prepared. The powdered mix was compacted into tablets of $250 \mathrm{mg}$ target weight at $10 \mathrm{Nm}^{-}$ 2 in the Manesty tableting machine (Shanghai, 
China) using the $10 \mathrm{~mm}$ punch and die set. The tablets produced were then kept for $24 \mathrm{~h}$ before evaluation to allow for elastic recovery.

\section{Evaluation of aspirin tablets prepared with co-processed excipients.}

Uniformity of weight. Ten (10) tablets were randomly selected and weighed on an analytical balance (Mettler Toledo, ME303E/02, USA) and the average weight was determined.

Tablet diameter and thickness. Diameter and thickness of ten (10) randomly selected tablets was determined using the micrometer screw gauge (Mitutoyo IDC-1012EB, Japan) and the average was computed.

Tablet hardness. Hardness ( $\mathrm{kgF}$ ) of five (5) randomly selected tablets was determined using the hardness tester (Erweka 65770 hardness tester, $\mathrm{GmbH}$, Germany) and the mean was calculated.

Tensile strength. Tablet diameter (d), thickness (t) and hardness (F) were used to compute the tensile strength (TS) according to the formula below [24];

Tensile strength $\left(\frac{N}{c m 2}\right) 2 F / \pi d t \quad \ldots \ldots$ Eqn 7

Friability test. Five (5) tablets were collectively weighed (W1), placed into the friabilator (Erweka 66939 Friabilator, $\mathrm{GmbH}$, Germany) and set to rotate at $25 \mathrm{rpm}$ for $4 \mathrm{~min}$. Afterwards, the tablets were de-dusted, reweighed (W2) and friability (\%) was calculated as;

$$
F(\%)=W 1-W 2 / W 1 \times 100 \quad \ldots \text { Eqn } 8
$$

Determination of wetting time. The method of $\mathrm{Bi}$ et al [25] was adopted with some modification. A tablet was placed into a petri dish containing $10 \mathrm{~mL}$ of water containing a green water-soluble dye at room temperature. The time taken for the green color to appear on the upper surface of the tablet was noted as the wetting time. Three (3) determinations of wetting time was made per batch then an average was computed.
Disintegration test. Six (6) tablets from each batch were placed in each of the six (6) compartments of the Disintegration tester (Erweka ZT4-4, Germany) containing distilled water maintained at $37 \pm 0.5^{\circ} \mathrm{C}$ as medium. The time taken for all the tablet particles to pass through the compartment's mesh was noted and the average was determined as the disintegration time.

In vitro dissolution studies. Calibration curve of aspirin was made using concentrations of (1 $\mu \mathrm{g}-500 \mu \mathrm{g})$, the corresponding absorbance was obtained from the UV-Visible Spectrophotometer (Agilent Cary 60, USA) at $275 \mathrm{~nm}$. One (1) tablet from each batch was placed in the dissolution basket and lowered into the dissolution vessel containing $0.1 \mathrm{~N}$ $\mathrm{HCl}(500 \mathrm{~mL})$ maintained at $37 \pm 0.5^{\circ} \mathrm{C}$. The apparatus (Dissolution tester, RC-6, India) was set to rotate at $50 \mathrm{rpm}$; aliquots of five $(5) \mathrm{mL}$ were withdrawn at intervals of 5, 10, 15, 20 min, filtered and thereafter replaced with equal volume of the medium. Absorbance of the withdrawn samples were determined at $275 \mathrm{~nm}$ using the UV-Visible spectrophotometer (Agilent Cary 60, USA) and the content of aspirin was determined using calibration curve.

Kinetics and mechanism of drug release. The kinetics of drug release was determined by fitting the data obtained from in vitro dissolution studies into the Zero order, First order, Higuchi Hixson-Crowell kinetic model while the mechanism of release was determined by the Korsmeyer-Peppas model [26]. The highest coefficient correlation was used to ascertain the best fit for modelling drug release.

\section{RESULTS AND DISCUSSION}

Flow properties of co-processed excipients. The flow properties of the co-processed excipients are presented in Table 3. It generally shows that the flow rate of CM2 $(4.10 \mathrm{~g} / \mathrm{sec})$ was better than those of CM3 $(0.26 \mathrm{~g} / \mathrm{sec})$ and CM1 $(0.14 \mathrm{~g} / \mathrm{sec})$. Angle of repose is a 
parameter that reflects the ability of a material to flow, values $<30^{\circ}$ implies excellent flow, 31 and $35^{\circ}$ portray good flow while 36 and 40 and those $>40^{\circ}$ signify fair and poor flow respectively [27]. The results show that all the prepared excipients possess excellent flow with values between 14 and $30{ }^{\circ}$. Correspondingly, all the excipients were found to have good propensity to decrease under pressure with Carr's index between 17 and 21 $\%$. The excipients were also found to be noncohesive as determined by the Hausner ratio values between 1.21 and 1.26 [28]. Moisture content plays an important role in product formulation viz-a-viz its stability, the presence of moisture in any formulation could lead to crystallization, loss of powder flow, loss of mechanical properties of the product in addition to promoting microbial growth during storage [29]. It is therefore important to ensure that the amount of moisture in any formulation is within specified limits. Table 3 shows CM1 and CM3 have inherent moisture content within the specifications of $15 \%$ for starchbased excipients [30] while CM2 had a higher value implying that more stringent storage conditions would be required for CM2.

Table 1: Composition for the preparation of co-processed excipients

\begin{tabular}{lccc}
\hline Batch & Ratio & Starch $(\mathrm{g})$ & Mannitol (g) \\
\hline CM1 & $1: 1$ & 15 & 15 \\
CM2 & $1: 2$ & 10 & 20 \\
CM3 & $2: 1$ & 20 & 10 \\
\hline
\end{tabular}

$C M 1=$ co-processed excipient containing Cyperus esculentus starch and mannitol in the ratio 1:1, CM2 = coprocessed excipient containing Cyperus esculentus starch and mannitol in the ratio 1:2; CM3 = co-processed excipient containing Cyperus esculentus starch and mannitol in the ratio 2:1

Table 2: Formula showing composition of ingredients for preparing Aspirin tablets (per tablet)

\begin{tabular}{lcccc}
\hline Ingredients/Batch & FM1 & FM2 & FM3 & FSG \\
\hline Aspirin (mg) & 75 & 75 & 75 & 75 \\
CM1 $(\mathrm{mg})$ & 12.5 & - & - & - \\
CM2 $(\mathrm{mg})$ & - & 12.5 & - & - \\
CM3 (mg) & - & - & 12.5 & - \\
SSG (mg) & - & - & - & 12.5 \\
MCC (mg) & qs & qs & Qs & Qs \\
Aerosil (mg) & 7.5 & 7.5 & 7.5 & 7.5 \\
Total $(\mathrm{mg})$ & 250 & 250 & 250 & 250 \\
\hline
\end{tabular}

$C M 1=$ co-processed excipient containing Cyperus esculentus starch and mannitol at 1:1 respectively; CM2 = co-processed excipient containing Cyperus esculentus starch and mannitol at 1:2 respectively; $C M 3=$ co-processed excipient containing Cyperus esculentus starch and mannitol at 2:1 respectively; $S S G=$ sodium starch glycolate; $F M 1=$ Tablets containing $5 \%$ w/w of CM1; FM2 = Tablets containing $5 \%$ w $/ w$ of CM2; FM3 = Tablets containing $5 \%$ w/w of CM3; FSS = Tablets containing $5 \%$ $w / w$ of $S S G$

Table 3: Some flow properties of the co-processed excipients

\begin{tabular}{lccc}
\hline Parameters/Batch & CM1 & CM2 & CM3 \\
\hline Flow rate $(\mathrm{g} / \mathrm{sec})$ & $0.14 \pm 0.00$ & $4.10 \pm 1.44$ & $0.26 \pm 0.06$ \\
Angle of repose $\left({ }^{\circ}\right)$ & $29.03 \pm 0.06$ & $14.36 \pm 0.05$ & $30.04 \pm 0.51$ \\
Carr's Index $(\%)$ & $17.31 \pm 0.90$ & $20.78 \pm 2.06$ & $19.07 \pm 1.51$ \\
Hausner ratio & $1.21 \pm 0.18$ & $1.26 \pm 0.00$ & $1.24 \pm 0.00$ \\
Moisture content $(\%)$ & 10.63 & 16.40 & 9.56 \\
\hline
\end{tabular}




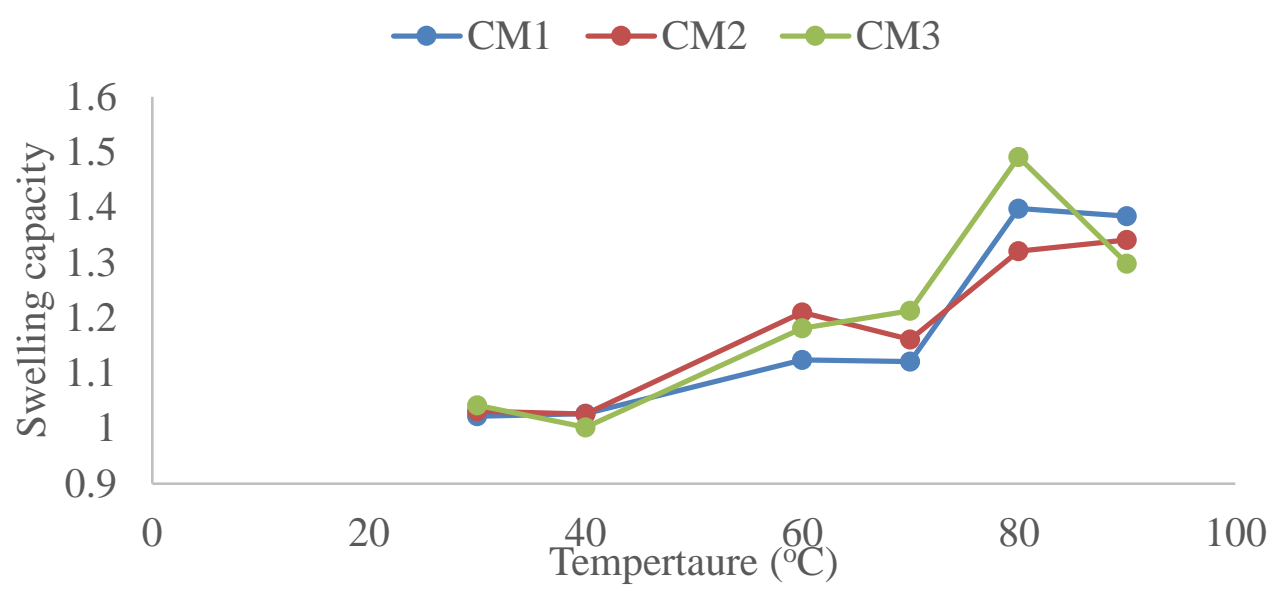

Figure 1: Swelling capacity of the co-processed excipients

Table 4: Some physical properties of Aspirin tablets formulated using the prepared co-processed excipients

\begin{tabular}{lllll}
\hline Parameter/Batch & FM1 & FM2 & FM3 & FSG \\
\hline Weight $(\mathrm{mg})$ & $240.7 \pm 0.00$ & $236.8 \pm 0.01$ & $237.3 \pm 0.00$ & $260.9 \pm 0.06$ \\
Thickness (mm) & $3.18 \pm 0.03$ & $3.17 \pm 0.03$ & $3.19 \pm 0.03$ & $3.21 \pm 0.15$ \\
Diameter (mm) & $10.04 \pm 0.04$ & $10.07 \pm 0.06$ & $10.06 \pm 0.05$ & $10.06 \pm 0.04$ \\
Hardness (kgF) & $7.08 \pm 0.41$ & $6.60 \pm 0.58$ & $5.64 \pm 0.30$ & $6.92 \pm 1.19$ \\
Tensile strength $\left(\mathrm{N} / \mathrm{cm}^{2}\right)$ & 14.12 & 13.17 & 11.32 & 14.03 \\
Friability (\%) & 0.26 & 0.42 & 0.34 & 0.25 \\
Wetting time (sec) & $34.33 \pm 0.05$ & $24.33 \pm 0.15$ & $9.00 \pm 0.05$ & $25.00 \pm 0.22$ \\
Disintegration time (min) & $6.49 \pm 1.69$ & $5.51 \pm 2.12$ & $4.26 \pm 3.19$ & $4.01 \pm 2.59$ \\
Dissolution efficiency t50 \% (min) & 5.12 & 4.34 & 3.64 & 3.16 \\
Dissolution efficiency t90 \% (min) & 29.84 & 18.60 & 14.24 & 9.78 \\
\hline
\end{tabular}

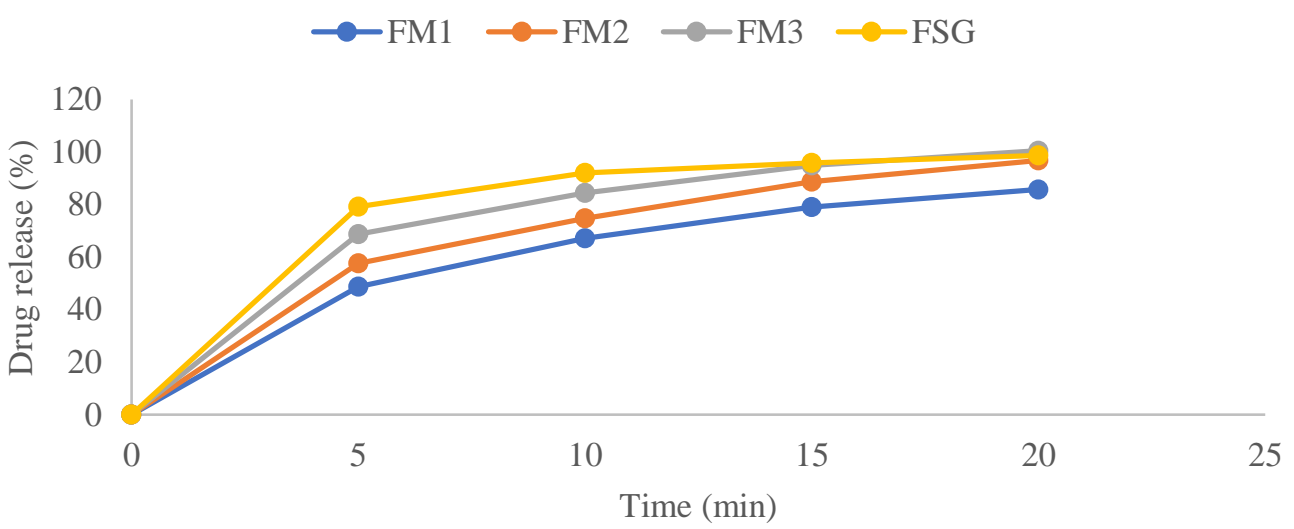

Figure 2: Dissolution profile of aspirin tablets prepared with the different co-processed excipient and sodium starch glycolate

Table 5: Kinetics and mechanism of aspirin tablet formulations prepared with co-processed excipeints

\begin{tabular}{lccccc} 
& Zero order & First order & Higuchi & \multicolumn{2}{c}{ Korsmeyer-Peppas } \\
\hline Formulations & $\mathrm{r}^{2}$ & $\mathrm{r}^{2}$ & $\mathrm{r}^{2}$ & $\mathrm{r}^{2}$ & $\mathrm{n}$ \\
\hline FM1 & 0.8610 & 0.2047 & 0.9918 & 0.5214 & 0.5586 \\
FM2 & 0.8500 & 0.0108 & 0.9891 & 0.1914 & 0.3416 \\
FM3 & 0.7715 & 0.0329 & 0.9587 & 0.8630 & 0.5554 \\
FSG & 0.6627 & 0.0180 & 0.8971 & 0.8411 & 0.5424 \\
\hline
\end{tabular}


Swelling capacity of co-processed excipients. Swelling capacity measures the ability of granules to absorb water and increase in size in the presence of heat. This parameter is also used to evaluate the behaviour of materials over a range of temperature as is applicable under industrial conditions. Figure 1 shows the swelling profile of the prepared excipients were similar although swelling was found to be slightly more appreciable in CM3 containing higher amount of starch. There was a general increase in swelling as temperature increased although no further increase was observed beyond $80^{\circ} \mathrm{C}$ after which there was no further increase in granule size. Swelling peak at $80^{\circ}$ $\mathrm{C}$ indicates that at this temperature, maximum interconnection between the polymer and water has been attained after which breakdown of the connection/link occurs [31]. This suggests that these excipients can be successfully employed in formulations at these temperatures $\left(30-80{ }^{\circ} \mathrm{C}\right)$.

Swelling is also a mechanism by which materials especially those containing starch, cause tablet disintegration and high swelling indices suggest that the materials would cause faster tablet disintegration [32]. This suggests the ability of the co-processed excipient; CM3 to effect faster tablet disintegration than CM2 or CM1.

Physical and mechanical properties of formulated tablets. The phyiscal properties of the formulated tablets are pressented in Table 4. The average weight of all tablets was found to be between 236 and $261 \mathrm{mg}$ which is within specified limits for tablets weighing $250 \mathrm{mg}$ [30]. The determination of uniformity of tablet weight is important because it ascertains that the ingredients are evenly distributed in each tablet thereby preventing problems such as inconsistencies in bioavailability of the active ingredient.

Tablet thickness and diameter are important considerations when packaging because tablets with varied measurements would affect the quantities specified for the final packaging vessel. The results presented in Table 4 show uniform tablet thickness (3.17 $3.21 \mathrm{~mm})$ and diameter (10.04 - $10.07 \mathrm{~mm})$. This can be attributed to the fact that the same punch and die set was used to compress the powder mix into tablets. Tablet thickness and diameter were also found to be within specified requirements of $\pm 5 \%$ of the average thickness and diameter [33].

Hardness is one of the parameters used to represent the mechanical strength of a tablet; it portrays the ability of the tablet to withstand the processes involved in its manufacturing, transportation, storage and use [34]. Uncoated tablets intended for immediate release have been reported to require hardness between 4 and $8 \mathrm{kgF}$ [35] although this can vary depending on the materials used in the formulation and intent of the formulation. Results in Table 4 show tablet hardness to be within this limit in the order FM1 $(7.08 \mathrm{kgF})>$ FSG (6.92 kgF)> FM2 (6.60 kgF) >FM3 (5.64 $\mathrm{kgF})$. Tablets prepared with the equal concentration of starch and mannitol (FM1) were found to be the strongest signifying that increasing the concentration of starch or mannitol in the co-processed excipient (FM2 and FM3 respectively) contributed to decreasing tablet strength. This was evidenced in the result of tensile strength as FM1 was observed to have the highest tensile strength (14.12 N/cm ${ }^{2}$ ) while FM3 had the least (13.12 $\mathrm{N} / \mathrm{cm}^{2}$ ). Although reports have demonstrated that high concentrations of starch disintegrants could have the ability to weaken tablet strength [36]. The results however show that there was no significant difference $(p>0.05)$ in tensile strength of all the tablets. Friability on the other hand measures the strength of the tablet via resistance to fracture and abrasion [37]. All the tablets had values between 0.25 and $0.42 \%$ which is $<1 \%$ specified as an acceptable limit [38].

The ability of a tablet to get wet is a precursor to its hydration and consequent 
break-up. Wetting time is the time taken for a tablet to take up fluid from its surrounding and the results show that they fell between 9.00 and $34.33 \mathrm{sec}$ (Table 4). Formulation FM3 containing the co-processed excipient with more of starch than mannitol $(2: 1)$ had the shortest wetting time $(9.00 \mathrm{sec})$ which was significantly different at $p<0.05$ from that of FM1 containing equal ratio of starch and mannitol $(34.33 \mathrm{sec})$ and those of FM2 and FSG although these later two were found to have similar wetting time (24.33 and 25.00 respectively). A requirement of period of 30 sec has been postulated for fast disintegrating excipients [39] and based on this, formulation FM1 containing equal amount of starch and mannitol does not meet this requirement. Faster wetting as observed in FM3 could be attributed to higher concentration of starch which enhanced porosity of the tablets and created a pathway for the liquid to be drawn into the tablet and unto the surface; it can also be linked to its swelling ability as shown in Figure 1. This process of wicking and capillary action is a known mechanism for some superdisintegrants [40].

Disintegration is the first visible change that is observed once a formulation comes in contact with a medium and determines the ability of a formulation to break-up and become soluble. Disintegration is often times linked with dissolution because it is assumed that the time taken for a tablet to disintegrate would indicate the time taken for the active ingredient housed therein to be available for bioavailability [41]. All the tablets were found to disintegrate between 4.01 and $6.49 \mathrm{~min}$ which is within the official specification for disintegration of immediate release uncoated tablets [30]. Disintegration time of FM3 (4.26 min) was found to be similar to that of the reference formulation (FSG; $4.01 \mathrm{~min}$ ) but significantly different at $\mathrm{p}$ $<0.05$ from that of FM2 (5.51 min) and FM2 (6.49 min). Shorter disintegration time of FM3 could be attributed to the composition of the excipient used in its formulation (starch to mannitol; 2:1) thereby causing rapid water uptake and breakdown of the particles holding the tablet together. This shows the effect of increased starch concentration in the prepared excipient and its influence in fast disintegration. Disintegrants are known to take up water and swell rapidly, creating a force within the tablet which overcomes the binding force between particles thereby causing the tablet to break up [42].

In vitro dissolution. Dissolution efficiency (t50 \%) as presented in Table 4 was found to be between 3.16 and $5.12 \mathrm{~min}$ with formulations FSG and FM3 showing the similar release rate $(3.12$ and 3.64 respectively) while FM1 released the same amount of drug $(50 \%)$ in $5.12 \mathrm{~min}$. The formulations were also found to release $90 \%$ (t90) between 9.78 and 24.84 min with FM1 showing release at the longest time while FSG showed the least time followed by FM3. The In vitro dissolution profile of aspirin tablets containing the different co-processed excipients are presented in Figure 2. Drug release at 5 min was found to be highest from FSG $(79.21 \%)$, while $68.73 \%$ was released from FM3 at same time and the least amount $(48.80 \%)$ was released from FM1.

Dissolution profile of FM3 was found to be comparable to that of tablets containing the sodium starch glycolate (FSG); this portrays the potential of FM3 as an excipient that can effect fast dissolution of tablet formulations. At the end of the dissolution study (20 min), formulation FM3 gave the highest release $(100 \%)$ followed by FSG (98.70 \%), FM2 (96.79 \%) and FM1 (85.75 $\%)$; suggesting its applicability in effecting fast in vitro dissolution. Generally, the results show the propensity of the co-processed excipients containing higher concentration of Cyperus esculentus starch than mannitol to cause fast drug release which can be attributed to the already discussed swelling, wetting and disintegration time. 
Release kinetics and mechanism of drug release. Release kinetics of the formulated tablets was determined by the Zero order, First order, Higuchi and Korsmeyer-Peppas models. Table 5 shows that Higuchi's model is the bestfit of all the models for the release of aspirin from the formulated tablets. This signifies that drug release was achieved by diffusion from the porous matrix system as a result of the contact of the tablets with the dissolution medium as postulated by Singhvi and Singh [43]. Mechanism of drug release as determined by Korsmeyer et al [44] recommends that when the exponent " $n$ " values $<0.5$ then drug release is diffusioncontrolled (Fickian) as is evident from formulation FM2 $(n=0.3416)$. On the other hand, values between 0.49 and 0.89 are postulated to indicate release controlled by swelling and diffusion (Non-fickian) as observed with formulations FM1, FM3 and FSG. Results from this study suggests that the drug release from tablets prepared with the coprocessed excipients was basically controlled by two and not just one process i.e. swelling and diffusion.

Conclusion. Co-processed excipients prepared from Cyperus esculentus starch and mannitol by fusion were found to influence tablet disintegration and in vitro dissolution. Incorporation of the co-processed excipients containing higher amount of Cyperus esculentus starch (CM3) in aspirin tablet formulation (FM3) showed faster wetting than tablets containing the reference disintegrant (FSG) while also having comparable disintegration time and dissolution profile with same reference formulation. This study shows that co-processing Cyperus esculentus starch and mannitol (2:1) by the fusion method can be employed in the manufacture fast dissolving tablet formulations.

\section{REFERENCES}

1. Kundusubrata, P. and Srinivasan, G. (2013). A Review on Co-Processed Excipients: A novel approach in formulation development. International Journal of Pharmaceutical Innovations, 3(5):25-41.

2. Reimerdes, D. (1993). The near future of tablet excipients; Manufacturing Chemist, 64(7):14-15.

3. Moreton, R.C. (1996). Tablet excipients to the year 2001: A look into the crystal ball; Drug Development and Industrial Pharmacy, 22:11-23.

4. Nachaegari S.K. and Bansal, A.K. (2004). Coprocessed excipients for solid dosage forms; Pharmaceutical Technology, 28:52-64.

5. Jacob, S.; Shirwaikar, A.A.; Joseph, A. and Srinivasan, K.K. (2007). Novel co-processed excipients of mannitol and microcrystalline cellulose for preparing fast dissolving tablets of glipizide. Indian Journal of Pharmaceutical Sciences, 69 (5): 633-639.

6. Ochubiojo, E.M. and Rodrigues, A. Starch: from food to medicine. In: Valdez B, Schorr M, Zlatev R, eds. Scientific, health and social aspects of the food industry. Rijeka: InTech, 355-80.

7. Anwar, E.; Komariah, E. and Junaedi, J. (2011). Preparation and characterization of co-processed excipient carrageenan-pregelatinized cassava starch propionate as a matrix in the gastroretentive dosage form; Makara Sains, 15(2):148-154.

8. Olowosulu, A.K.; Oyi, A.; Isah, A.B. and Ibrahim, M.A. (2011). Formulation and evaluation of novel coprocessed excipients of maize starch and acacia gum (starac) for direct compression tableting; International Journal of Pharmaceutical Research and Innovation, 2:39-45, 2011.

9. Ogunjimi, A.T. and Alebiowu, G. (2013). Flow and consolidation properties of neem gum co-processed with two pharmaceutical excipients; Powder Technology, 246:187-192.

10. Adeoye, O. and Alebiowu, G. (2014). Evaluation of co-processed disintegrants produced from tapioca starch and mannitol in orally disintegrating paracetamol tablet; Acta Poloniae Pharmaceutical, 71(5):803-811.

11. Mshelia, J.G.; Apeji, Y.E. and Olayemi, O.J. (2015). Powder, compaction and tableting properties of co-processed silicified starch; Journal of Pharmaceutical Research International, 6(2):131-140.

12. Adetunji, O.A. and Odeniyi, M.A. (2016). Material and compression properties of Cedrela 
odorata gum co-processed with plantain starch and microcrystalline cellulose; Polymers in Medicine, 46(1):35-43.

13. Apeji, Y.; Aluga, D.; Olayemi, O.; Oparaeche, C.; Anyebe, S.; Gamlen, M. and Oyi, A. (2017). Comparative analysis of co-processed starches prepared by three different methods; British Journal of Pharmacy, 2(1):55-59.

14. Channarong, S.; Thiptinnakorn, T. and Chuetee, P. (2017). Development of mannitol-corn starch co-processed direct compression excipient using lactitol as binder; Isan Journal of Pharmaceutical Sciences, 13(4):63-76.

15. Okunlola, A. (2018). Flow, compaction and tabletting properties of co-processed excipients using pregelatinized Ofada rice starch and HPMC; Journal of Excipients and Food Chemistry, 9 (1):4-15.

16. Narayan, P. and Hancock, B.C. (2003). The relationship between the particle properties, mechanical behaviour and surface roughness of some pharmaceutical excipient compacts. Material Sciences Engineering, 355:24-36.

17. Rowe, R.C.; Sheskey, P.J. and Quin, M.E. (2012). Handbook of Pharmaceutical Excipients. 7th edition. London: Pharmaceutical Press; 2012. 479482.

18. Ohrem, H.L.; Schornick, E. and Ognibene, R. (2014). Why is mannitol becoming more and more popular as a pharmaceutical excipient in solid dosage forms?; Pharmaceutical Development and Technology, 19(3):257-262.

19. Azubuike, C.P.; Fabiyi, R.O.; Oseni, B.A. and Igwilo, C.I. (2019). Evaluation of disintegrant potential of carboxymethyl starch derived from Cyperus esculentus (Cyperaceae) tubers. Tropical Journal of Natural Products Research, 3:246-251

20. Builders, P.F.; Anwunobi, P.A.; Mbah, C.C. and Adikwu, M.A. (2013). New direct compression excipient from tigernut starch: physicochemical and functional properties, AAPS Pharmaceutical Sciences Technology, 14:818-827.

21. Onyinye, D.O.; Azubuike, C.P.; Aloko, S.; Ologunagba. M.O. and Igwilo, C.I. (2019).Characterization and disintegrant potential of phosphorylated tiger nut (Cyperus esculentus) starch in immediate release ibuprofen tablet formulation. Dhaka University Journal of Pharmaceutical Sciences, 18:21-29.

22. Kunle, O.O.; Ibrahim, Y.E.; Emeje, M.O.; Shada, S. and Kunle, Y. (2003). Extraction and physicochemical compaction properties of Tacca starch- a Potential Pharmaceutical excipient; Starch/Starke, 55:319-325.

23. Cooper, J. and Gunn, C. (1986). Powder flow and compaction. In: Carter SJ, editor. Tutorial Pharmacy. New Delhi, India: CBS Publishers and Distributors; pp. 211-233.

24. Fell, J.T. and Newton, J.M. (1970). Determination of tablet strength by the diametral compression test; Journal of Pharmaceutical Sciences, 59:688-691.

25. Bi, Y.; Sunada, H.; Yonezawa, Y.; Danjo, K.; Otsuka, A. and Lida, K. (1996). Preparation and evaluation of compressed tablet rapidly disintegrating in the oral cavity; Chemical and Pharmaceutical Bulletin, 44:2121-2127.

26. Siepmann, J.; Streubel, A. and Peppas, N.A. (2002). Understanding and predicting drug delivery from hydrophilic matrix tablets using the sequential layer model. Pharmaceutical Research, 19:306-314.

27. Mohammadi, M.S. and Harnby, N. (1997). Bulk density modelling as a means of typifying the microstructure and flow characteristics of cohesive powders; Powder Technology, 92:1-8.

28. Panda, D.; Choudhury, N.S.K.; Yedukondalu, M.; Si, S. and Gupta, R. (2008). Evaluation of gum of Moringa oleifera as a binder and release retardant in tablet formulation; Indian Journal of Pharmaceutical Sciences, 70(5):614-618.

29. Adane, M.; Endale, A.; Bultosa, G.; AbdelMohsen, M.G. and Gebre-Mariam, T. (2006). Isolation and physicochemical characterization of Godare (Colocasia esculenta) starch from Ethiopia; Ethiopian Pharmaceutical Journal, 24:13-22.

30. British Pharmacopoeia (2002). Volume I and II: Her Majesty's Stationery Office, University Press, Cambridge, London, UK.

31. Rojas, J.; Guisao, S. and Ruge, V. (2012). Functional assessment of four types of disintegrants and their effect on the spironolactone release properties; AAPS Pharmaceutical Science Technology, 13(4):1054-1062.

32. Colombo, P.; Conte, U.; Caramella, C.; Geddo, M. and La Manna, A. (1984). Disintegrating force as a new formulation parameter; Journal of Pharmaceutical Sciences, 73(5):701-705.

33. Chaturvedi, M.; Kumar, M.; Pathak, K.; Bhatt, S. and Saini, V. (2017). Surface solid dispersion and solid dispersion of meloxicam: comparison and product development; Advances in Pharmaceutical Bulletin, 7(4):569-577. 
34. Odeku, O.A. and Itiola, O.A. (2003). Effects of interacting variables on the tensile strength and the release properties of paracetamol tablets; Tropical Journal of Pharmaceutical Research, 2(1):147-153.

35. Quodbach, J.; Moussavi, A.; Tammer, R.; Frahm, J. and Kleinebudde, P. (2014). Tablet disintegration studied by high-resolution real-time magnetic resonance imaging; Journal of Pharmaceutical Sciences, 103:249-255.

36. Odeku, O.A. and Alabi, C.O. (2007). Evaluation of native and modified forms of Pennisetum glaucum (millet) starch as disintegrant in chloroquine tablet formulations; Journal of Drug Delivery Science and Technology, 17(2):155-157.

37. Itiola, O.A.; Odeniyi, M.A. and Adetunji, O.A. (2006). Compression, mechanical and release properties of chloroquine phosphate tablets containing corn and trifoliate yam starches as binders; Tropical journal of pharmaceutical research, 5 (2):589-596.

38. Banker, G.S. and Anderson, N.R. (1986).Tablet. In: Lachman, L., Lieberman, H.A. and Kanig, J.L. (eds), The Theory and Practice of Industrial Pharmacy, 3rd edn. Lea and Febiger, Philadelphia, pp. 301-303.

39. Rehman, A.; Bushra, R.; Beg, A.E.; Ali, H.; Zafar, F.; Ashfaq, M.; Alam, S.; Mustapha, O. and
Shafique, S. (2018). Effects of superdisintegrants in oral dissolving formulation of cinitapride tablets; Pakistan Journal of Pharmaceutical Sciences, 31(2):643-650.

40. Gajare, G.G.; Bakliwal, S.R.; Rane, B.R.; Gujrathi, N.A. and Pawar, S.P. (2011). Mouth dissolving tablet: A Review; International Journal of Pharmaceutical Research and Development, 6:280296.

41. Parma, B.; Baria, H.; Tank, M. and Faldu, D. (2009). Formulation and evaluation of domperidone fast dissolving tablets; International Journal of Pharmaceutical Technology and Research, 1(3):483487.

42. Liberman, H.A.; Lachman, L. and Schawstr, J.B. (1989). Pharmaceutical dosage forms: Tablets. Vol.2.

43. Singhvi, G. and Singh, M. (2011). In-vitro drug release characterization models; International Journal of Pharmaceutical Sciences and Research, 2:77-84.

44. Korsmeyer, R.W.; Gurny, R.; Doelker, E.; Buri, P. and Peppas, N.A. (1983). Mechanisms of solute release from porous hydrophilic polymers; International Journal of Pharmaceutics, 15:25-35. 